\title{
A robust version of the Lee filter for speckle reduction and contrast enhancement applied to side scan sonar images
}

\author{
$1^{\text {er }}$ Viviana E. Ferraggine \\ Departamento de Computacion y Sistemas \\ INTIA - Facultad de Cs. Exactas UNICEN \\ Tandil, Argentina \\ vferra@exa.unicen.edu.ar
}

\author{
$2^{\text {nd }}$ Sebastian A. Villar \\ CIFICEN - UNICEN - CICPBA - CONICET \\ INTIA - Facultad de Cs. Exactas UNICEN \\ Olavarria, Argentina \\ svillar@fio.unicen.edu.ar
}

\begin{abstract}
Sonar images are typically affected by a granular pattern interference known as speckle noise, which degrades image contrast. To aid in object detection and recognition for speckled imagery, a robust version of the Lee filter is presented. The new method essentially combines robust statistics with an adaptive approach to achieve an effective balance between contrast stretching and speckle reduction. Tests were performed on real sonar images, where objective metrics and direct visual perception were used to evaluate the results. Experiments have shown that this easy-to-implement filter remarkably highlights edges and details with apparent speckle reduction, offering a promising simple tool that may be useful in segmentation and classification applications.
\end{abstract}

Index Terms-Side Scan Sonar, noise reduction, image enhancement, robust statistics.

\section{INTRODUCCIÓN}

Los dispositivos acústicos proporcionan imágenes de alta resolución en áreas submarinas, incluso en aguas con escasa o nula visibilidad [1]. Estos dispositivos son relevantes para numerosas aplicaciones prácticas, como geología marina, pesca comercial, arqueología submarina, búsqueda de recursos, extracción y perforación de petróleo, inspección y mantenimiento de tuberías, detección de minas o desechos y otros tipos de monitoreo [2], [3]. En este sentido, un sonar de barrido lateral (SSS, Side Scan Sonar) representa una herramienta eficaz para el mapeo de alta resolución del lecho marino debido a su madurez tecnológica y a una excelente relación costo/calidad [2]. Los SSS utilizan arreglos lineales de transductores emitiendo y recibiendo en fase uno con otro, o en una relación de fase controlada. En cada ciclo de adquisición de datos, el SSS escanea hacia abajo y a ambos lados (babor y estribor) en forma de abanico perpendicular a la dirección de avance del vehículo que transporta el sonar. Los pulsos acústicos viajan por la columna de agua, impactan en el fondo del mar, y, a continuación, el eco o señal de retrodispersión retorna al sensor de recepción donde se cuantifica su amplitud. Los datos adquiridos son proyectados sobre una línea de escaneo (beam). De este modo, una imagen de SSS se conforma colocando una secuencia de beam consecutivos a lo largo de la dirección de navegación del sonar para crear una representación del lecho marino en 2D [2], [4], [5]. Sin embargo, las imágenes de SSS presentan la característica de bajo rango dinámico de los valores de intensidad debido a la pérdida de transmisión acústica, la reverberación y el entorno hidrológico complejo. Un bajo rango dinámico imposibilita la distinción de los objetos de estudio y detalles en las imágenes de SSS, y por lo tanto dificulta la automatización de las aplicaciones prácticas ya mencionadas. A su vez, las imágenes de SSS presentan un ruido inherente y característico conocido con el nombre de speckle. Este ruido es común en los sensores remotos que utilizan radiaciones coherentes como fuente; cuando se emite un pulso en forma de ondas acústicas, luego de la interacción con el lecho marino estas ondas ya no están en fase. Se producen así interferencias positivas o destructivas, que generan retornos anormalmente altos o bajos. El ruido speckle es difícil de distinguir automáticamente, y su eliminación completa es compleja. En la actualidad no existe un algoritmo universal para el mejoramiento de imágenes acústicas de SSS, aunque se tienen diversas estrategias cuyos resultados varían de acuerdo al dispositivo sonar utilizado, la longitud de onda, la profundidad de adquisición, el tiempo de procesamiento, la calidad resultante y demás [2]. La elección de un método de reducción de ruido speckle generalmente depende de los requerimientos específicos para una aplicación en particular; sin embargo, muchas capacidades de reducción de ruido deterioran la resolución de la imagen (por ejemplo, provocando la pérdida de detalles y bordes difusos). Al procesar imágenes de SSS con la presencia de ruido speckle, la reducción de ruido y el estiramiento del contraste (contrast stretching) deben equilibrarse para preservar las características relevantes de los objetos de estudio. En la literatura de las últimas décadas, se han introducido numerosas alternativas de pre-procesamiento para eliminar el ruido speckle y mejorar el contraste de imágenes de SSS que abarcan diferentes niveles de complejidad que influyen notablemente en su etapa posterior de post-procesamiento, como por ejemplo en la detección y reconocimiento de objetos [6], [7]. Entre los métodos de pre-procesamiento de imágenes acústicas se encuentran el método variacional (variational 
approach) [8], representación escasa (sparse representation) [9], corrección de histograma (histogram Correction) [10] y estiramiento no lineal (nonlinear stretching) [11], transformada Curvelente (curvelent transform) [12], transformada Wavelet (wavelet transform), entre otros [13]. Los métodos detallados implican numerosos pasos secuenciales, utilizan técnicas combinadas, procedimientos complejos iterativos, establecen umbrales o parámetros subjetivos y además, requieren frecuentemente una cierta cantidad de información previa para obtener una imagen resultante de calidad. En este contexto, se ha sugerido en el trabajo de [14] que un enfoque de reducción de ruido speckle ideal debería combinar las ventajas del filtro de mediana para la preservación de la estructura y velocidad en tiempo de cómputo, y la simplicidad y eficacia conceptual del filtro Lee [15]. En este contexto, este trabajo presenta un enfoque adaptativo de una combinación estadísticamente robusta del filtro de Lee para lograr un equilibrio efectivo entre el estiramiento del contraste y la reducción del ruido speckle, y que además resulta de un método verdaderamente simple de implementar. El trabajo se organiza de la siguiente forma: en la Sección I se presenta nuestra propuesta del filtro de Lee Robusto, en la Sección II se presentan los resultados experimentales utilizando diferentes imágenes reales de SSS con métricas de evaluación objetivas y una directa percepción visual. Por ultimo, se presentan las conclusiones obtenidas y futuros trabajos.

\section{Filtro de LeE Robusto}

El filtro de Lee realiza una combinación lineal de la intensidad observada y el valor de intensidad media local dentro de una ventana fija de radio $\mathrm{r}$; si $x(i, j)$ es el valor de píxel que se está procesando, ubicado en la fila $i$ y la columna $j$ de la imagen, este trabajo propone generar un valor de pixel de salida $z(i, j)$ calculado según la siguiente ecuación:

$z(i, j)=m_{e}[W(i, j)]+\frac{\operatorname{IQR}[W(i, j)]}{\operatorname{IQR}[\operatorname{Imagen}]}\left(x(i, j)-m_{e}[W(i, j)]\right)$

donde $W(i, j)$ denota el conjunto de valores de la ventana cuadrada centrada en $x(i, j)$, mientras que $m_{e}[A]$ y $I Q R[A]$ indican respectivamente la mediana y el rango intercuartil para el conjunto de valores de dicha ventana (matriz A).

En el lado derecho de la ecuación (1), el primer término revela que el nuevo método emplea por defecto un filtro de mediana para reducir el ruido speckle. A su vez, el último término es el responsable del estiramiento del contraste.

El $I Q R$ local se calcula como la diferencia entre la mediana de los valores más altos que el $50 \%$ de los píxeles y la mediana de los valores más bajos que el $50 \%$ de los píxeles para cada ventana; en todos los casos se siguió un cálculo de la mediana eficiente utilizando el método discutidos en [16]. Tanto el $I Q R$ de la ventana actual como el de la imagen completa representan indicadores robustos del rango dinámico local y global, respectivamente. En cada paso de filtrado, su relación se utiliza para controlar el valor de salida: cuando se procesan áreas homogéneas (el $\operatorname{IQR}[W(i, j)]$ o local representa un valor mucho más estrecho que el IQR Imagen] o global), esta relación se acerca a cero y prevalece el filtro de mediana. En cambio, cuando se procesan áreas con texturas ( $I Q R$ local representa un valor mucho más amplio que el $I Q R$ global) esta relación aumenta y la diferencia inicial entre el valor de píxel actual y la mediana local se estira. Como resultado, los bordes y los detalles se agudizan mientras que el patrón granular ruidoso de los niveles de gris se reduce no solo en áreas homogéneas sino en general.

\section{RESUlTAdos EXPERIMENTALES}

La reducción de ruido speckle de varias imágenes de SSS utilizando el algoritmo propuesto se evalúa y compara con el filtro Lee y el filtro de mediana. Para una evaluación cuantitativa del método presentado se seleccionaron cuatro imágenes de libre acceso de SSS como se muestran en la Figura 1 y primer columna de la Figura 3.

A cada imagen de prueba se le aplicaron los tres métodos utilizando una ventana cuadrada con tamaños de radio que varían de 1 a 10. Los tres métodos se implementaron en código C++ bajo el entorno de desarrollo integrado Nokia QtCreator para GNU/Linux utilizando las estructuras de datos eficientes de OpenCV 2.3. Los experimentos se realizaron en una PC equipada con una CPU Intel® CoreTM i7-3630QM de 2,4 $\mathrm{GHz}$ y $8 \mathrm{~Gb}$ de RAM con sistema operativo Ubuntu 14.04 LTS (32 bits).

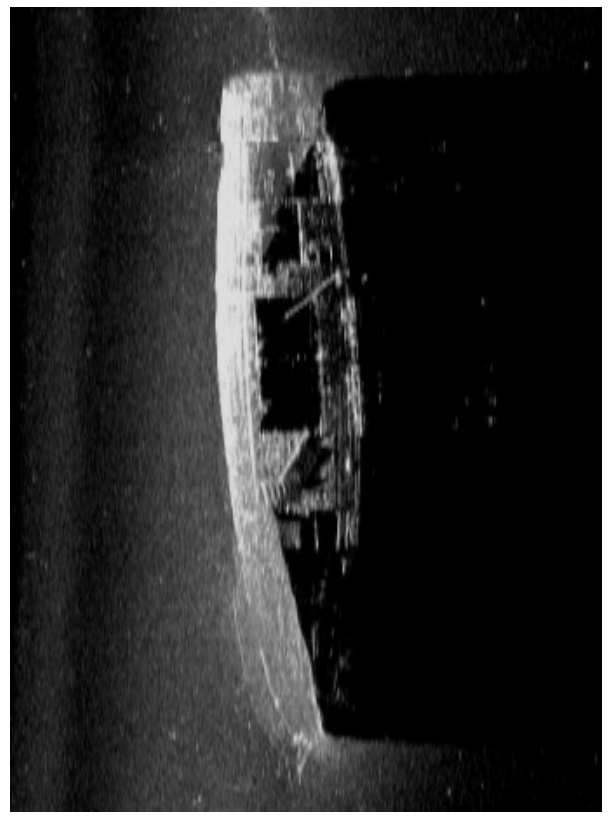

Fig. 1: Imagen de SSS que muestra las características de un barco hundido.

Se utilizó la percepción visual herramienta sencilla para evaluar la calidad de los resultados, dado que permite tanto la detección de aquellas características que representan el comportamiento de un método de reducción de ruido como su capacidad de preservar bordes y objetos, el grado de 
desenfoque resultante y la posible presencia de artefactos que apenas se detectan mediante una precisa observación.

Para realizar una comparación cuantitativa entre el método propuesto y los de referencia (filtro de mediana y filtro de Lee), se seleccionaron cuatro métricas full-reference y una métrica no-reference que son consistentes con las evaluaciones visuales subjetivas: proporción máxima de señal a ruido (PSNR, Peak Signal to Noise Ratio), error absoluto de histogramas normalizados (NAE, Normalized histograms Absolute Error), índice medio de semejanza estructural (MSSIM, Mean Structural Similarity Index) e índice de contraste, y puntuación de calidad espacial de imagenes sin referencia (BRISQUE, Blind Referenceless Image Spatial Quality Evaluator). Cabe señalar que las métricas PSNR, MSSIM y NAE no se encuentran correlacionadas entre sí, mientras que MSSIM implica el cálculo del índice de contraste.

En la Fig. 1 se muestra una imagen de SSS de un barco hundido que se encontró en el Lago de Washington a una profundidad de 200 metros adquirida a una frecuencia de $600 \mathrm{kHz}$ en un rango de 50 metros. Estas imágenes fueron descargadas desde el sitio [17].

Analizando la Fig. 2, se pueden observar varios patrones. Si se toman como referencia los resultados con radio de ventana 1, las salidas de los tres métodos parecen bastante similares. Sin embargo, el rango dinámico ampliado alcanzado por el método propuesto ya se percibe en el brillo y la nitidez del borde izquierdo y los detalles en el armazón del barco. A partir del radio 3, la limpieza del fondo marino alcanzada por la mediana y el filtro Lee estándar mejora gradualmente, pero degrada los detalles característicos del barco; el desenfoque es mayor para el filtro de mediana, mientras que un halo parece crecer fuera del borde izquierdo para el filtro Lee. El filtro de Lee Robusto, a su vez, alcanza un nivel similar de limpieza en el fondo del mar y las finas estructuras del casco del barco no se pierden sino que se destacan, mientras que los bordes se afilan. En general, la menor degradación visual de la imagen original se logra con el nuevo método cuando aumenta el radio; y también es notable una salida bastante estable independientemente del tamaño de ventana.

En términos de la evaluación cuantitativa para las Fig. 2 y 3 (resultados detallados en la Tabla I) se observa que, cuando se compara con el filtro de mediana y el filtro Lee estándar, el filtro propuesto muestra una mejor relación señal ruido (valores de PSNR 15\% y 5\% más altos en promedio, respectivamente), mejor preservación de la información estructural (valores MSSIM 16\% y 11\% más altos en promedio, respectivamente), mejor contraste (valores de contraste $16 \%$ y $10 \%$ más altos en promedio, respectivamente), menor error absoluto comparando el histograma normalizado con el de la imagen original (valores de NAE 9\% y 30\% más bajos en promedio, respectivamente) y una calidad percibida más alta (valores BRISQUE 16\% y 19\% más bajos en promedio, respectivamente).

La Fig. 3 muestra los resultados de aplicar el filtro de mediana, el filtro de Lee y el filtro propuesto para un radio de ventana de tamaño 3 sobre imágenes SSS. Estas imágenes fueron descargadas desde el sitio [17] y son de especial interés en estudios de rescate, arqueológicos o detección de fisuras en partes de desagües.

En la fila superior se tiene un barco hundido encontrado en la Bahía Elliot, Washington a 250 metros de profundidad, imagen adquirida a una frecuencia de $600 \mathrm{kHz}$ en un rango de 50 metros. Como se puede apreciar los tres métodos parecen hacer frente de manera uniforme al ruido speckle, pero la versión del filtro propuesto ofrece relieves más nítidos y bordes bien definidos sobre la estructura del barco.

En la fila del centro se tienen identificados numerosos carros de carbón que se encontraron en el Lago de Washington a 200 metros de profundidad, imagen adquirida a una frecuencia de $600 \mathrm{kHz}$ en un rango de 20 metros. Según la referencia estos carros de carbón se cayeron desde una barcaza. En esta imagen se puede apreciar como el filtro propuesto preserva las características de cada carro de carbón como así también de los pequeños objetos reflectantes alrededor de los mismos. Tanto el filtro de Lee como el filtro de mediana produce una imagen resultante difusa que no conserva las características del objeto de estudio.

En la fila inferior se tiene una tubería de desagüe, imagen adquirida a una frecuencia de $600 \mathrm{kHz}$ en un rango de 50 metros. En estos resultados todas las métricas tienden a favorecer el filtro de Lee Robusto (Tabla I), que exhibe un efecto verdaderamente notable en base a la calidad percibida resultante. Nuevamente, el filtro propuesto muestra que se conserva las características del desagüe y no produce una imagen borrosa como presentan el filtro de mediana y el filtro de Lee.

\section{CONCLUSIONES}

En este articulo se presentó un método verdaderamente simple y efectivo para reducir ruido speckle y estirar el contraste. Los resultados experimentales han demostrado que la versión que se propone a partir del filtro Lee supera a alternativas similares y convencionales que poseen un mayor nivel de complejidad sobre la base de pasos iterativos, o información previa, o técnicas combinadas, o estableciendo parámetros con umbrales subjetivos.

Además, este trabajo espera impulsar estrategias que ahorren recursos de procesamiento, demostrando que métodos que siguen resoluciones simples también pueden lograr buenos resultados. La investigación futura explorará la selección automática de valores de cuartiles utilizados para el cálculo de $I Q R$, con el fin de determinar si es posible una mejor manera de capturar el rango dinámico local mientras se controlan los valores de ruido. Las imágenes sintéticas también se incluirán en nuevos análisis.

Adicionalmente, se aplicará el método presentado a imágenes de láser, SAR y ultrasonido que también sufren el deterioro producto del ruido speckle. 


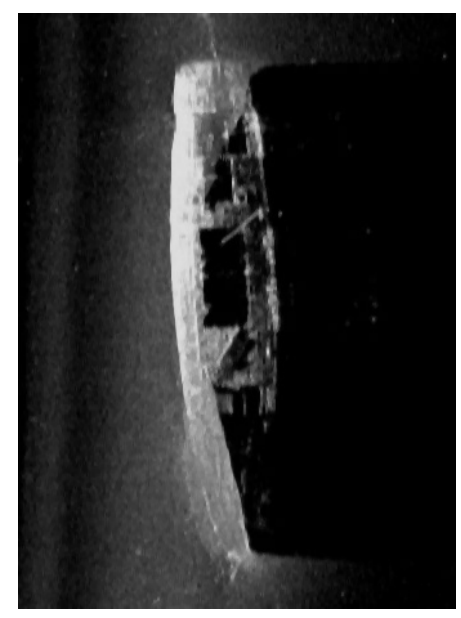

(a) Median filter, radius 1

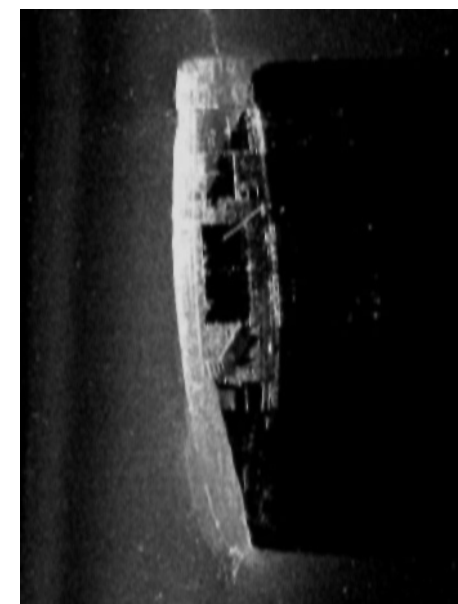

(e) Lee filter, radius 1

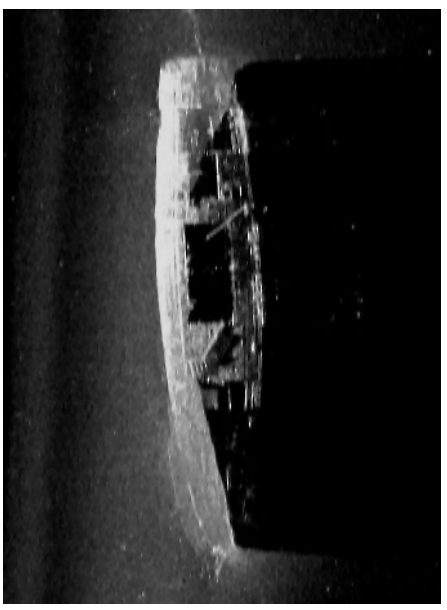

(i) Robust Lee filter, radius 1

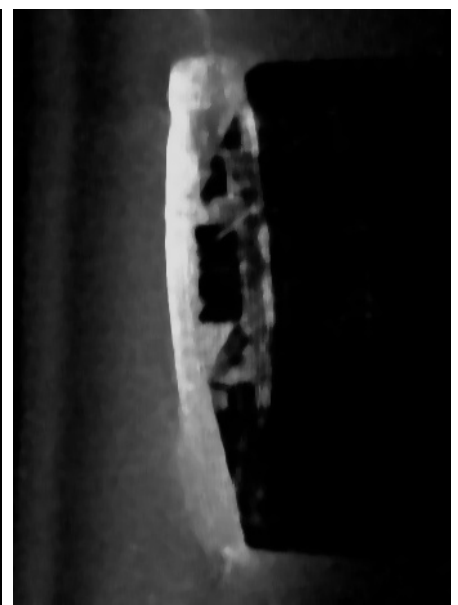

(b) Median filter, radius 3

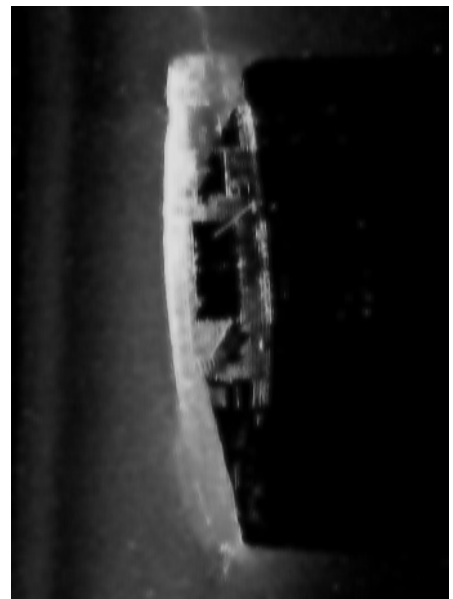

(f) Lee filter, radius 3

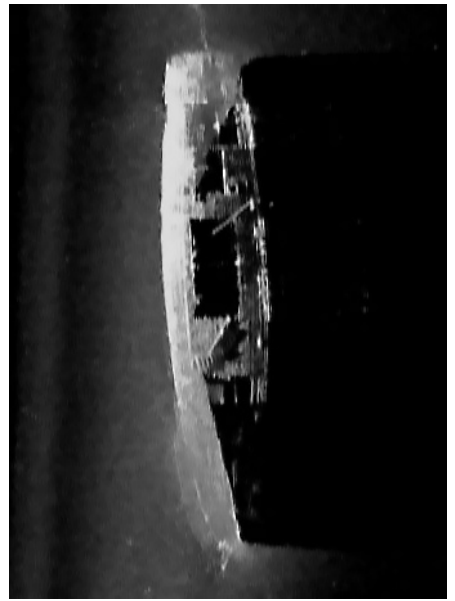

(j) Robust Lee filter, radius 3

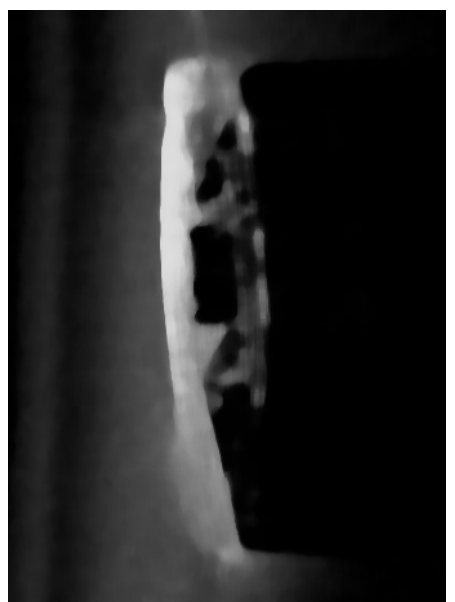

(c) Median filter, radius 5

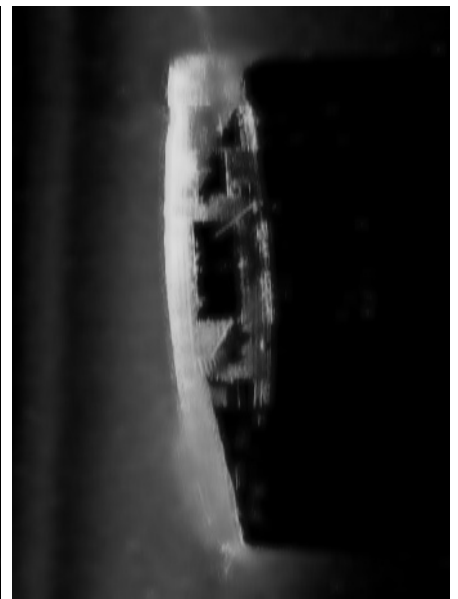

(g) Lee filter, radius 5

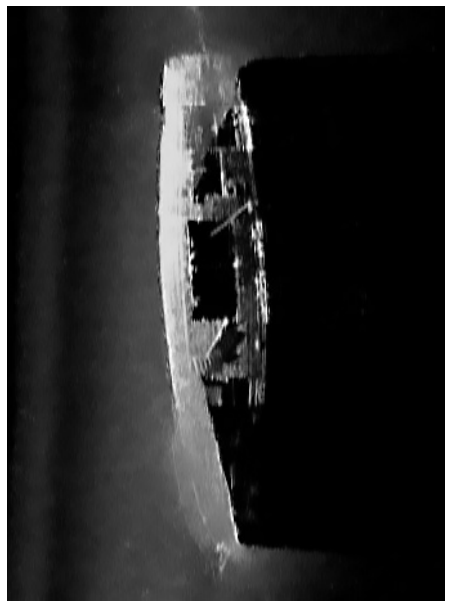

(k) Robust Lee filter, radius 5

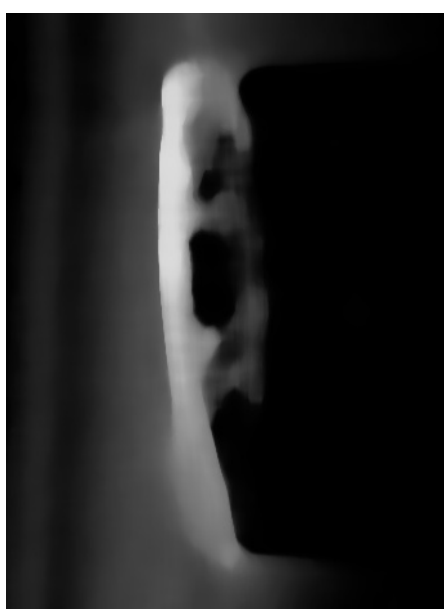

(d) Median filter, radius 10

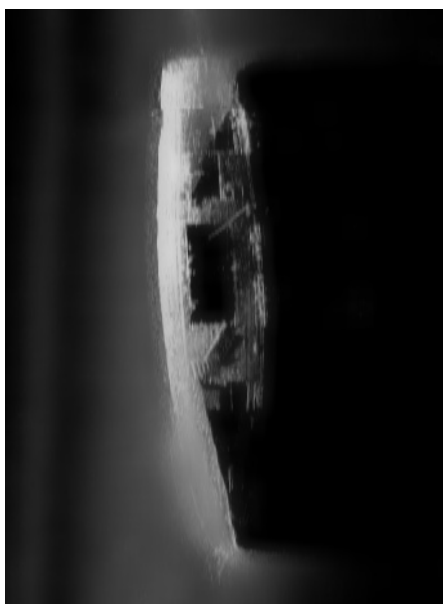

(h) Lee filter, radius 10

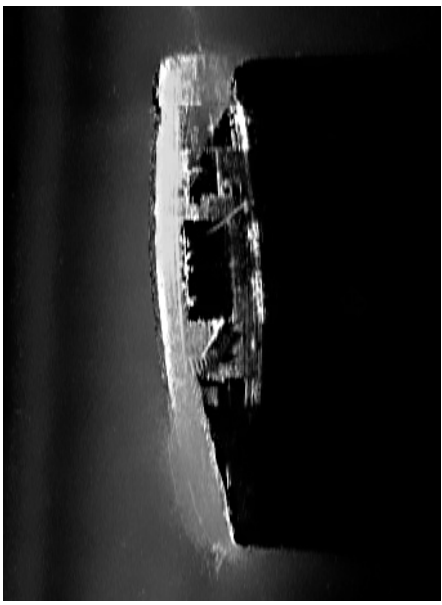

(1) Robust Lee filter, radius 10

Fig. 2: Imagen de SSS de un barco hundido. Se muestran los resultados del filtro de mediana (fila superior), el filtro Lee estándar (fila central) y del filtro Lee Robusto (fila inferior) para ventanas de diferentes tamaños de radio. 

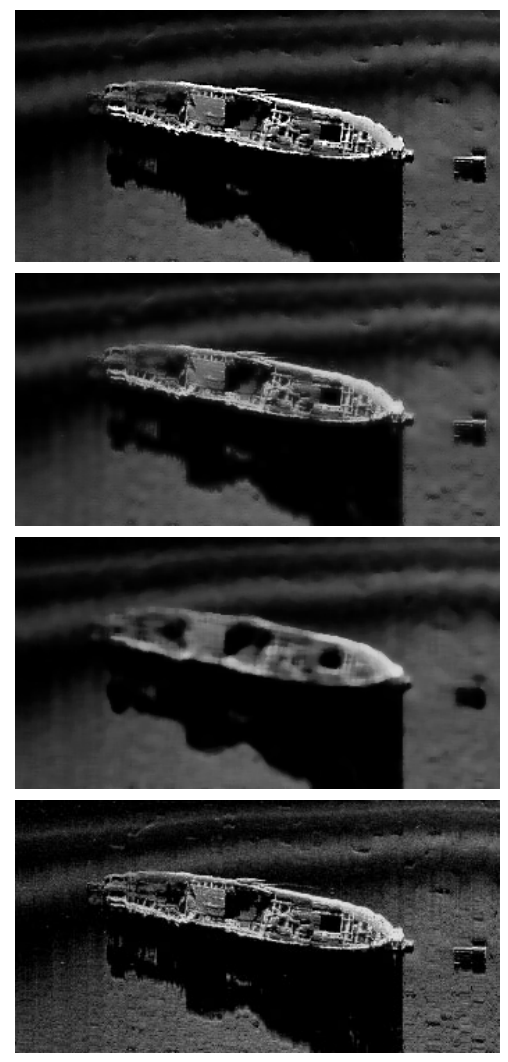
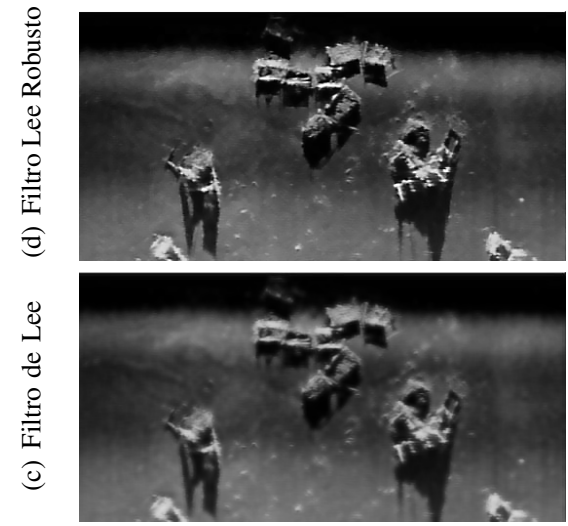

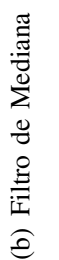

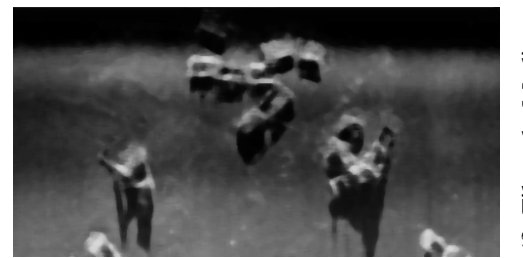

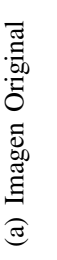

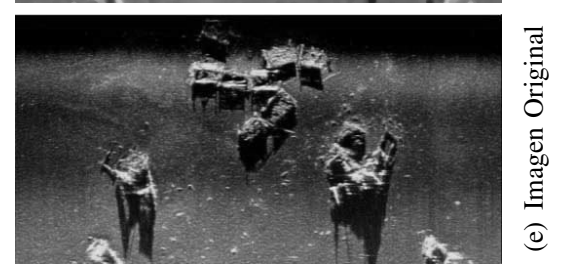

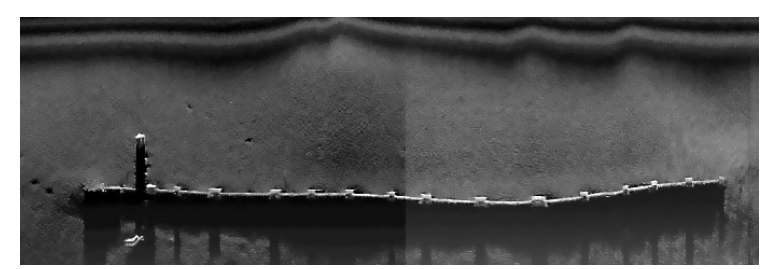
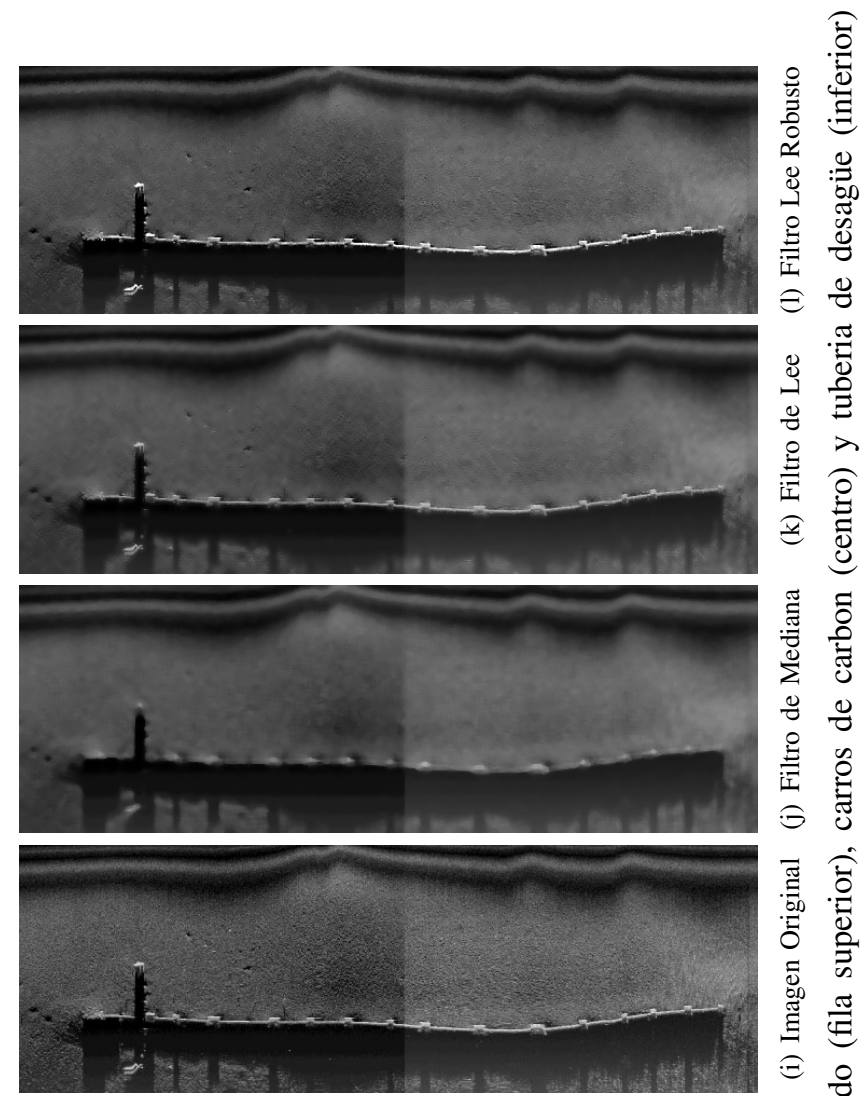

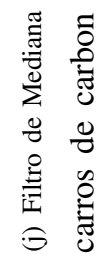

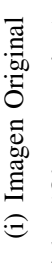

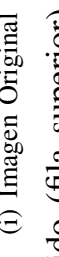

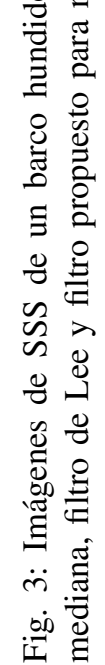


TABLE I: Comparación cuantitativa del filtro de mediana $(M F)$, filtro Lee $(L F)$ y filtro de Lee Robusto $(R L F)$. Se muestran los valores de varias métricas objetivas ( en negrita el mejor resultado) para imágenes reales de SSS para ventanas de diferentes tamaños. Las métricas utilizadas son PSNR (peak signal-to-noise ratio), MSSIM (Mean Structural Similarity), NAE (Normalized Absolute Error), Constrast y BRISQUE (Blind Referenceless Image Spatial Quality Evaluator).

\begin{tabular}{|c|c|c|c|c|c|c|c|c|c|c|c|c|c|c|c|c|c|}
\hline \multirow[b]{2}{*}{ Imagen } & \multirow[b]{2}{*}{$r$} & \multicolumn{3}{|c|}{ PSNR } & \multicolumn{2}{|c|}{ MSSIM } & \multirow{2}{*}{$\frac{\uparrow}{R L F}$} & \multicolumn{3}{|c|}{ NAE } & \multicolumn{2}{|c|}{ Contrast } & \multirow{2}{*}{$\frac{\uparrow}{R L F}$} & \multicolumn{4}{|c|}{ BRISQUE $\quad \downarrow$} \\
\hline & & $M F$ & $L F$ & $R L F$ & $M F$ & $L F$ & & $M F$ & $L F$ & $R L F$ & $M F$ & $L F$ & & $M F$ & $L F$ & $R L F$ & Orig. \\
\hline \multirow[t]{5}{*}{ Fig.2 } & 1 & 31.11 & 32.80 & 35.36 & 0.85 & 0.87 & 0.92 & 0.06 & 0.07 & 0.05 & 0.86 & 0.87 & 0.93 & 34.54 & 36.32 & 36.27 & 43.44 \\
\hline & 2 & 28.80 & 31.12 & 33.58 & 0.78 & 0.81 & 0.88 & 0.08 & 0.09 & 0.07 & 0.78 & 0.82 & 0.89 & 34.81 & 36.53 & 31.53 & \\
\hline & 3 & 27.82 & 30.38 & 32.52 & 0.75 & 0.79 & 0.87 & 0.09 & 0.12 & 0.08 & 0.76 & 0.79 & 0.88 & 40.25 & 46.12 & 32.81 & \\
\hline & 5 & 26.52 & 29.57 & 30.65 & 0.72 & 0.76 & 0.86 & 0.12 & 0.16 & 0.10 & 0.73 & 0.78 & 0.87 & 50.34 & 51.37 & 37.95 & \\
\hline & 10 & 24.55 & 28.50 & 28.54 & 0.69 & 0.75 & 0.87 & 0.14 & 0.23 & 0.14 & 0.71 & 0.79 & 0.88 & 54.64 & 48.31 & 35.70 & \\
\hline \multirow[t]{5}{*}{ Fig.3 [a)..d)] } & 1 & 25.24 & 28.55 & 30.13 & 0.72 & 0.79 & 0.86 & 0.12 & 0.16 & 0.09 & 0.73 & 0.80 & 0.86 & 23.33 & 32.47 & 25.87 & 37.36 \\
\hline & 2 & 23.12 & 27.46 & 28.73 & 0.61 & 0.72 & 0.82 & 0.16 & 0.19 & 0.11 & 0.62 & 0.73 & 0.83 & 43.83 & 41.55 & 27.80 & \\
\hline & 3 & 22.10 & 26.96 & 27.66 & 0.55 & 0.70 & 0.81 & 0.18 & 0.24 & 0.13 & 0.57 & 0.71 & 0.82 & 50.90 & 38.51 & 24.31 & \\
\hline & 5 & 20.75 & 26.35 & 26.25 & 0.49 & 0.67 & 0.80 & 0.21 & 0.31 & 0.16 & 0.51 & 0.70 & 0.82 & 54.06 & 35.80 & 19.69 & \\
\hline & 10 & 18.94 & 25.33 & 25.27 & 0.43 & 0.62 & 0.82 & 0.32 & 0.42 & 0.21 & 0.46 & 0.71 & 0.84 & 53.16 & 37.99 & 29.46 & \\
\hline \multirow[t]{5}{*}{ Fig.3 [e)..h)] } & 1 & 31.52 & 32.40 & 35.31 & 0.87 & 0.88 & 0.92 & 0.09 & 0.11 & 0.07 & 0.87 & 0.88 & 0.92 & 30.68 & 34.90 & 31.30 & 23.05 \\
\hline & 2 & 24.25 & 29.28 & 31.31 & 0.67 & 0.73 & 0.83 & 0.12 & 0.16 & 0.11 & 0.67 & 0.73 & 0.84 & 36.08 & 42.93 & 34.42 & \\
\hline & 3 & 23.32 & 28.26 & 30.02 & 0.59 & 0.69 & 0.81 & 0.15 & 0.18 & 0.14 & 0.60 & 0.69 & 0.81 & 43.62 & 40.42 & 20.15 & \\
\hline & 5 & 21.73 & 27.25 & 27.02 & 0.53 & 0.66 & 0.79 & 0.19 & 0.19 & 0.16 & 0.54 & 0.67 & 0.80 & 54.83 & 40.38 & 23.64 & \\
\hline & 10 & 20.21 & 26.07 & 24.02 & 0.48 & 0.66 & 0.79 & 0.25 & 0.24 & 0.20 & 0.49 & 0.69 & 0.80 & 60.98 & 31.99 & 19.66 & \\
\hline \multirow[t]{5}{*}{ Fig.3 [i)..1)] } & 1 & 26.65 & 28.98 & 30.67 & 0.61 & 0.73 & 0.84 & 0.13 & 0.15 & 0.11 & 0.61 & 0.73 & 0.84 & 24.29 & 25.64 & 23.81 & 31.54 \\
\hline & 2 & 25.42 & 28.20 & 30.09 & 0.51 & 0.67 & 0.82 & 0.18 & 0.17 & 0.14 & 0.51 & 0.67 & 0.82 & 36.05 & 29.05 & 13.13 & \\
\hline & 3 & 24.85 & 27.93 & 29.61 & 0.48 & 0.65 & 0.81 & 0.20 & 0.18 & 0.14 & 0.48 & 0.66 & 0.82 & 46.90 & 27.74 & 14.47 & \\
\hline & 5 & 24.14 & 27.50 & 29.60 & 0.44 & 0.65 & 0.82 & 0.23 & 0.21 & 0.15 & 0.45 & 0.65 & 0.83 & 52.20 & 22.52 & 20.54 & \\
\hline & 10 & 22.80 & 26.66 & 29.73 & 0.38 & 0.65 & 0.85 & 0.31 & 0.25 & 0.16 & 0.39 & 0.67 & 0.85 & 56.96 & 25.82 & 25.80 & \\
\hline
\end{tabular}

\section{REFERENCES}

[1] T. Celik and T. Tjahjadi, "A Novel Method for Sidescan Sonar Image Segmentation," IEEE Journal Oceanic Engineering, vol. 36, no. 2, pp. 186-194, Apr. 2011.

[2] X. Lurton, An Introduction to Underwater Acoustics: Principles and Applications, NY, USA: Springer-Verlag, 2002.

[3] P. Chapple, "Automated Detection and Classification in High-Resolution Sonar Imagery for Autonomous Underwater Vehicle Operations," Maritime Operations Division Defense Science and Technology Organization, Edinburgh South Australia, DSTO-GD-0537, 2008.

[4] G. G. Acosta and S. A. Villar, "Accumulated CA-CFAR Process in 2-D for Online Object Detection From Sidescan Sonar Data," IEEE Journal Oceanic Engineering, vol. 40, no. 3, pp. 558-569, Jul. 2015.

[5] P. Blondel, The Handbook of Sidescan Sonar, Praxis Publishing. UK: Springer Berlin Heidelberg, ch. 3 and 4, 2009.

[6] S. A. Villar, M. De Paula, F. J. Solari and G. G. Acosta, "A framework for acoustic segmentation using order statistic-constant false alarm rate in two dimensions from sidescan sonar data," IEEE Journal of Oceanic Engineering, vol. 43 (3), 735-748, 2018.

[7] S. A. Villar, B. V. Menna, S. Torcida and G. G. Acosta, "Efficient approach for OS-CFAR 2D technique using distributive histograms and breakdown point optimal concept applied to acoustic images," IET Radar, Sonar and Navigation, vol. 13, (12), pp. 2071-2082, 2019.

[8] D. Chen, X. Chu, F. Ma and X. Teng, "A variational approach for adaptive underwater sonar image denoising," 4th International Conference on Transportation Information and Safety (ICTIS), pp. 1177-1181, 2017.

[9] D. Wu, X. Du and K. Wang, "An Effective Approach for Underwater Sonar Image Denoising Based on Sparse Representation," IEEE 3rd International Conference on Image, Vision and Computing (ICIVC), pp. 389-393, 2018.

[10] T. Arici S. Dikbas and Y. Altunbasak, "A Histogram Modification Framework and Its Application for Image Contrast Enhancement," IEEE Transactions on Image Processing vol. 18 (9), pp. 1921-1935, Sept. 2009.

[11] T. Celik and T. Tjahjadi, "Automatic Image Equalization and Contrast Enhancement Using Gaussian Mixture Modeling," IEEE Transactions on Image Processing vol. 21 (1), pp. 145-156, Jan. 2012.

[12] Y. Zhou L. Qingwu and H. Guanying, "Automatic Side-Scan Sonar Image Enhancement in Curvelet Transform Domain," Mathematical Problems in Engineering pp. 1-14, 2015.
[13] R. Priyadharsini T. Sree Sharmila and V. Rajendran, "A wavelet transform based contrast enhancement method for underwater acoustic images," Multidimensional Systems and Signal Process vol. 29 (4), pp. 1845-1859, 2017.

[14] A. Lopes, E. Nezry, R. Touzi and H. Laur, "Structure detection and statistical adaptive speckle filtering in SAR images," International Journal of Remote Sensing, vol. 14, pp. 1735-1758, 1993.

[15] J. S. Lee, "Speckle analysis and smoothing of synthetic aperture radar images,"Computer Graphics and Image Processing, vol. 17, pp. 24-32, Sept. 1981.

[16] S. A. Villar, S. Torcida and G. G. Acosta, "Median filtering: a new insight," Journal of Mathematical Imaging and Vision, vol. 58, pp. 130146, Dec. 2016.

[17] [Online]. Available: https://www.innerspaceexploration.org/ 